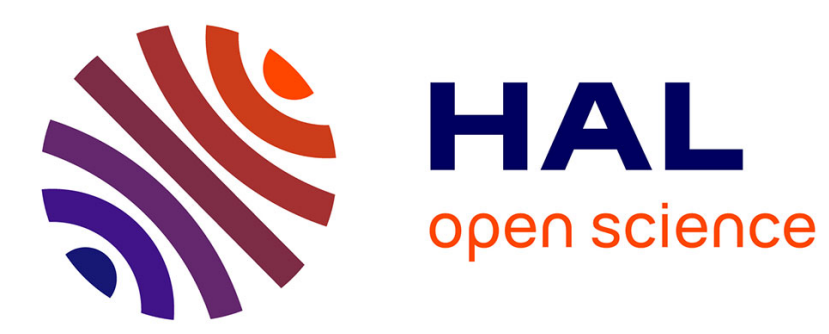

\title{
Profils de température à l'intérieur d'un modèle sphéroïdal de tumeur soumis à une onde électromagnétique hyperfréquence
}

\author{
R. Deleuil, R. Zimmer
}

\section{- To cite this version:}

R. Deleuil, R. Zimmer. Profils de température à l'intérieur d'un modèle sphéroïdal de tumeur soumis à une onde électromagnétique hyperfréquence. Revue de Physique Appliquée, 1988, 23 (7), pp.12651271. 10.1051/rphysap:019880023070126500 . jpa-00245940

\author{
HAL Id: jpa-00245940 \\ https://hal.science/jpa-00245940
}

Submitted on 1 Jan 1988

HAL is a multi-disciplinary open access archive for the deposit and dissemination of scientific research documents, whether they are published or not. The documents may come from teaching and research institutions in France or abroad, or from public or private research centers.
L'archive ouverte pluridisciplinaire HAL, est destinée au dépôt et à la diffusion de documents scientifiques de niveau recherche, publiés ou non, émanant des établissements d'enseignement et de recherche français ou étrangers, des laboratoires publics ou privés. 


\title{
Profils de température à l'intérieur d'un modèle sphéroïdal de tumeur soumis à une onde électromagnétique hyperfréquence
}

\author{
R. Deleuil ( $\left.{ }^{1}\right)$ et R. Zimmer $\left({ }^{2, *}\right)$ \\ (1) Département de Radioélectricité, Université de Provence, Marseille, France \\ (2) Fondation de Recherche en Hormonologie, Fresnes, France
}

(Reçu le 19 octobre 1987, accepté le 25 janvier 1988)

\begin{abstract}
Résumé. - On a modélisé une tumeur par un ellipsoïde de révolution allongé d'indice complexe et on a cherché une solution au problème de l'interaction d'une onde électromagnétique avec un tel objet afin d'évaluer les effets thermiques qui peuvent en résulter. De la connaissance du champ interne on a déduit la densité de puissance électromagnétique dissipée dans l'objet; ensuite, on a résolu le problème thermique associé et, enfin, on a déterminé l'évolution de la température dans le modèle en fonction de ses caractéristiques physiques et de la durée d'exposition au champ micro-onde incident. Par référence aux valeurs rencontrées à $2450 \mathrm{MHz}$ pour les paramètres électromagnétiques et thermiques de tissus sains et cancéreux, on présente des cartes de la température en tout point du modèle tumoral.
\end{abstract}

\begin{abstract}
To represent a tumor, a prolate spheroid model has been chosen. To calculate the power deposition due to a microwave incident field the problem of the scattering of a linearly polarized electromagnetic plane wave by a homogeneous prolate spheroid with a complex index of refraction has been solved. From the internal field the power density inside the spheroid is deduced and the heat conduction problem related to the same object is then treated. With the values of thermic and electromagnetic parameters taken at $2450 \mathrm{MHz}$ both for sound and cancerous tissues, various maps of the temperature inside this spheroidal model are given.
\end{abstract}

\section{Introduction.}

L'emploi de l'hyperthermie dans le traitement des tumeurs humaines a été largement débattu au cours des quinze dernières années. L'importance de la valeur de la température atteinte dans la tumeur visà-vis de celle existant dans les tissus environnants ainsi que l'allure de la répartition interne de la température ont été soulignées, tout comme d'ailleurs, l'intérêt de faire appel aux micro-ondes pour provoquer l'échauffement recherché. En fait, dans le cas de tumeurs cancéreuses localisées et superficielles, l'hyperthermie micro-onde, qui permet un traitement loco-régional, peut être avantageusement associée à la chimiothérapie ou à la radiothérapie dont elle en potentialise les effets [17].

L'utilisation de l'hyperthermie en clinique nécessite la mesure et le contrôle de la température en tout point de l'ensemble composite tumeur-tissu

(*) Adresse actuelle : Hoffmann-La Roche \& Co. LTD, Basle, Switzerland. sain ; cela paraît maintenant possible grâce à divers dispositifs ou procédés (microsondes placées in vivo, thermographie infrarouge, radiométrie micro-onde) qui limitent les effets traumatisants sur le patient.

Mais à côté de ces considérations pratiques, on peut aussi envisager une approche théorique à ce type de problème en cherchant à calculer l'énergie électromagnétique dissipée à l'intérieur et dans le proche voisinage d'une tumeur, puis en déterminant l'évolution de la température au sein de l'ensemble. La résolution de ce problème comporte deux phases (l'une électromagnétique, l'autre thermique) et demande, au préalable, l'adoption d'un modèle adéquat de tumeur.

En ce qui concerne les tumeurs mammaires, sur lesquelles notre intérêt s'est porté, il est tout à fait réaliste d'adopter un modèle sphéroïdal allongé. Cependant, compte tenu des fréquences appliquées (quelques $\mathrm{GHz}$ ) et des dimensions caractéristiques de la tumeur (quelques $\mathrm{cm}$ ), il n'était pas envisageable d'avoir recours à des approximations lors du traitement de la première phase. La résolution, sous 
la forme la plus générale, du problème de l'interaction d'une onde électromagnétique avec un sphéroïde diélectrique présente une certaine complexité mais, offre l'avantage d'ouvrir un champ d'applications débordant largement du cadre ayant suscité la présente étude puisque ce problème intéresse l'optique, l'astrophysique, les télécommunications, le génie biomédical, ...

\section{Etapes de l'étude.}

On considère un ellipsoïde de révolution allongé, homogène, de demi-axes $a$ et $b(a>b)$, taillé dans un matériau diélectrique à pertes $\left(\varepsilon_{2}, \sigma_{2} \neq 0\right.$, $\mu_{2} \simeq \mu_{0}$ ), noyé dans un diélectrique quasiment parfait $\left(\varepsilon_{1}, \sigma_{1} \simeq 0, \mu_{2} \simeq \mu_{0}\right)$ et recevant, sous une incidence quelconque $\zeta$ (mesurée par rapport au grand axe $a$ ), une onde électromagnétique, harmonique, plane, polarisée rectilignement (Fig. 1). On distingue les cas TM et TE suivant que le champ électrique incident ${ }^{\mathrm{i}} \mathbf{E}$ appartient ou est perpendiculaire au plan d'incidence $\mathrm{O} x z$ ). Ainsi défini, on reconnaît un problème de diffraction électromagnétique dans le domaine de résonance et concernant un objet connexe d'indice complexe.

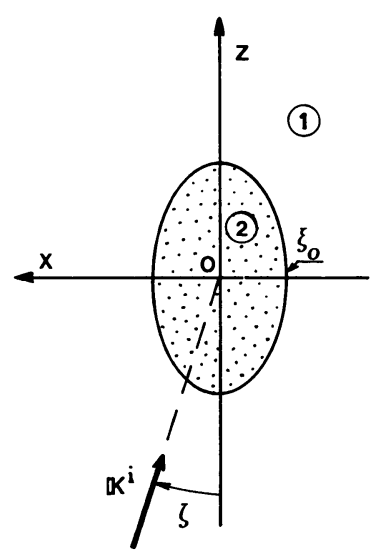

Fig. 1. - Géométrie du problème.

[Geometry of the problem.]

Au champ incident sont associés un champ externe et un champ interne qui génèrent des effets thermiques dans les deux milieux. Si ces effets sont liés à la géométrie, ils dépendent surtout des valeurs des conductivités électriques $\left(\sigma_{1}\right.$ et $\left.\sigma_{2}\right)$ et thermiques $\left(k_{1}\right.$ et $\left.k_{2}\right)$ des milieux en présence. Préalablement à toute investigation théorique (et éventuellement expérimentale), il est nécessaire de s'assurer que les valeurs de ces paramètres ne vont pas à l'encontre du but recherché, c'est-à-dire, n'interdisent pas de provoquer, au sein du tissu cancéreux, un échauffement préférentiel susceptible de le rendre plus sensible à une radiothérapie ou à une chimiothérapie ultérieure.
Cette hypothèque étant levée, il faut alors traiter un problème de conduction de la chaleur, depuis un sphéroïde porteur d'une distribution interne de sources, vers un milieu extérieur supposé d'étendue infinie. On peut alors, en fonction de la durée d'exposition au champ électromagnétique appliqué, calculer la température en tout point du sphéroïde et de son proche voisinage et, ensuite, jouer sur les paramètres géométriques (incidence $\zeta$, rapport $a / b)$, électromagnétiques $\left(\varepsilon_{i}, \sigma_{i}\right)$ et thermiques $\left(k_{i}\right)$ pour déterminer leur influence sur la distribution de la température dans les régions d'intérêt.

\section{Traitement théorique.}

Dans le système de coordonnées de l'ellipsoïde de révolution allongé, $0 \xi \eta \phi$ (Fig. 2), la surface de l'objet, qui coïncide avec l'une des surfaces de coordonnées $\xi=\xi_{0}$, partage l'espace en deux régions (1 et 2 ) dans lesquelles la partie spatiale $\mathbf{F}_{i}(i=1,2)$, de tout vecteur caractéristique du champ électromagnétique satisfait l'équation :

$$
\Delta \mathbf{F}_{i}+K_{i}^{2} \mathbf{F}_{i}=0
$$

avec :

$$
K_{i}^{2}=\omega^{2} \varepsilon_{i} \mu_{i}+j \omega \sigma_{i} \mu_{i} .
$$

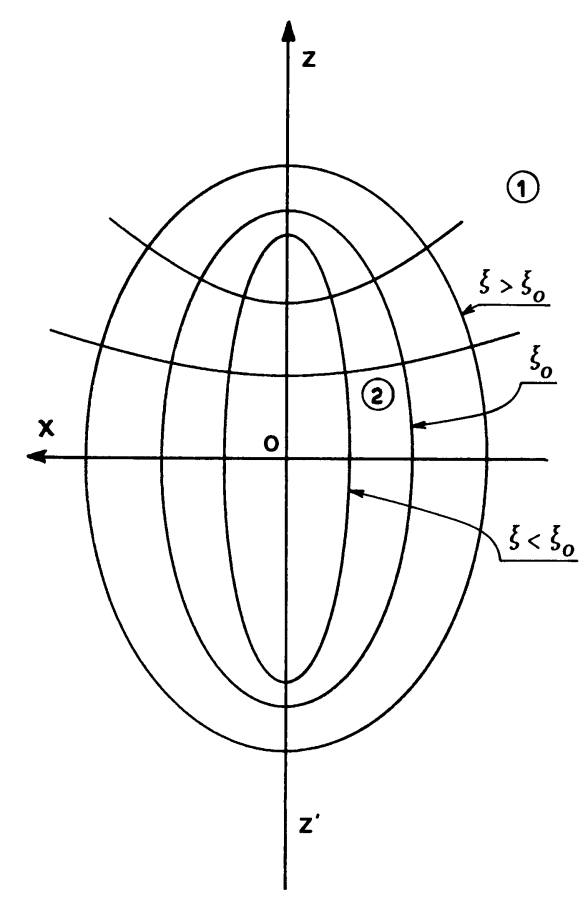

Fig. 2. - Système de coordonnées de l'ellipsoïde de révolution allongé.

[Prolate spheroidal coordinates.]

Dans chacune de ces régions les vecteurs du champ peuvent s'exprimer à l'aide des fonctions d'onde vectorielles $\mathbf{M}$ et $\mathbf{N}$ [8], telles que $\mathbf{M}=\operatorname{rot}(\mathbf{a} \psi)$ et 
$K_{i} \mathbf{N}=\operatorname{rot} \mathbf{M}$, où a est tout vecteur constant (ou le vecteur position $\mathbf{r}$ ) et $\psi$ est une fonction scalaire solution de l'équation :

$$
\Delta \psi+K_{i}^{2} \psi=0
$$

Cette équation est séparable et $\psi$ s'obtient à partir de produits du type :

$$
\begin{aligned}
& \psi_{m n}^{(h)}(c ; \xi, \eta, \varphi)=R_{m n}^{(h)}(c ; \xi) S_{m n}^{(1)}(c ; \eta) \times \\
& \times \sin _{\cos }^{\cos } m \phi, \quad h=1,2,3,4
\end{aligned}
$$

où $c=K_{2} d$, avec $d=\sqrt{a^{2}-b^{2}}=$ demi-distance interfocale, $R$ et $S$ désignant les fonctions sphéroïdales radiales et angulaires [9].

Dans le cas TM, par exemple, on a les expressions suivantes pour les vecteurs champs électriques incident ${ }^{\mathrm{i}} \mathbf{E}_{1}$, diffusé ${ }^{\mathrm{s}} \mathbf{E}_{1}$ et réfracté ${ }^{\mathrm{t}} \mathbf{E}_{2}$ :

$$
\begin{aligned}
& { }^{\mathrm{i}} \mathbf{E}_{1}=\sum_{m, n} j^{n}\left[f_{m n}(\xi) \mathbf{M}_{\mathrm{o}, m n}^{r(1)}-j g_{m n}(\xi) \mathbf{N}_{\mathrm{e}, m n}^{r(1)}\right] \\
& { }^{\mathrm{s}} \mathbf{E}_{1}=\sum_{m, n} j^{n}\left[\alpha_{m n} \mathbf{M}_{\mathrm{o}, m n}^{r(3)}-j \beta_{m n} \mathbf{N}_{\mathrm{e}, m n}^{r(3)}\right] \\
& { }^{\mathrm{t}} \mathbf{E}_{2}=\sum_{m, n} j^{n}\left[\gamma_{m n} \mathbf{M}_{\mathrm{o}, m n}^{r(1)}-j \delta_{m n} \mathbf{N}_{\mathrm{e}, m n}^{r(1)}\right]
\end{aligned}
$$

Des expressions similaires sont obtenues pour les vecteurs champs magnétiques. Les coefficients inconnus $\alpha_{m n}, \beta_{m n}, \gamma_{m n}$ et $\delta_{m n}$ sont déterminés en exprimant la continuité des composantes tangentielles de $\mathbf{E}$ et $\mathbf{H}$ à la surface $\boldsymbol{\xi}=\boldsymbol{\xi}_{0}$.

On calcule ensuite les champs électromagnétiques externe (ou diffusé ${ }^{\mathrm{s}} \mathbf{E}_{1}$ ) et interne (ou réfracté ${ }^{t} \mathbf{E}_{2}$ ) puis, de ce dernier, on déduit la densité de puissance dissipée dans le sphéroïde, soit :

$$
P_{2}(\mathbf{r})=\left.\sigma_{2}\right|^{\mathrm{t}} \mathbf{E}_{2} \cdot{ }^{\mathrm{t}} \mathbf{E}_{2 *} \mid / 2 \text {. }
$$

Bien que le champ interne soit le seul intéressant pour l'objectif visé, le champ externe a été également évalué. Ce faisant, il est possible, d'une part, de disposer des résultats concernant le problème canonique extérieur de la diffraction d'une onde électromagnétique par un ellipsoïde de révolution allongé d'indice complexe, et d'autre part, de valider nos traitements théoriques et numériques en considérant le cas particulier d'un diffuseur diélectrique parfait et en comparant alors nos résultats à ceux obtenus par Asano et Yamamoto [10] puis par Schaefer [11]. Ces auteurs, qui se sont intéressés à la diffusion de la lumière par des hydrométéores ou par des particules interplanétaires, ont calculé le champ diffusé à grandes distances $\left({ }^{\mathrm{s}} \mathbf{E}_{1}\right)$ par un modèle sphéroïdal allongé de particule ; ils ont ensuite déterminé, dans le cas particulier d'une incidence axiale, les intensités $i_{1}(\theta)$ et $i_{2}(\theta)$ des composantes (respectivement perpendiculaire et parallèle au plan de diffusion) de l'intensité diffusée dans la direction $\theta$, pour $\theta \in[0, \pi]$.

A titre de vérification, on a considéré un ellipsoïde de révolution allongé, caractérisé par un rapport $a / b=2$, un paramètre $c=K_{2} d=5$ (où $K_{2}=$ $2 \pi / \lambda$ et $d=\sqrt{a^{2}-b^{2}}$, un indice de réfraction réel $n_{2}=1,33$ et recevant sous incidence axiale $(\zeta=0)$ une onde plane. Les courbes $i_{1}(\theta)$ et $i_{2}(\theta)$, obtenues dans ces conditions (Fig. 3 ), sont pratiquement indiscernables de celles données par Asano et Yamamoto [10].

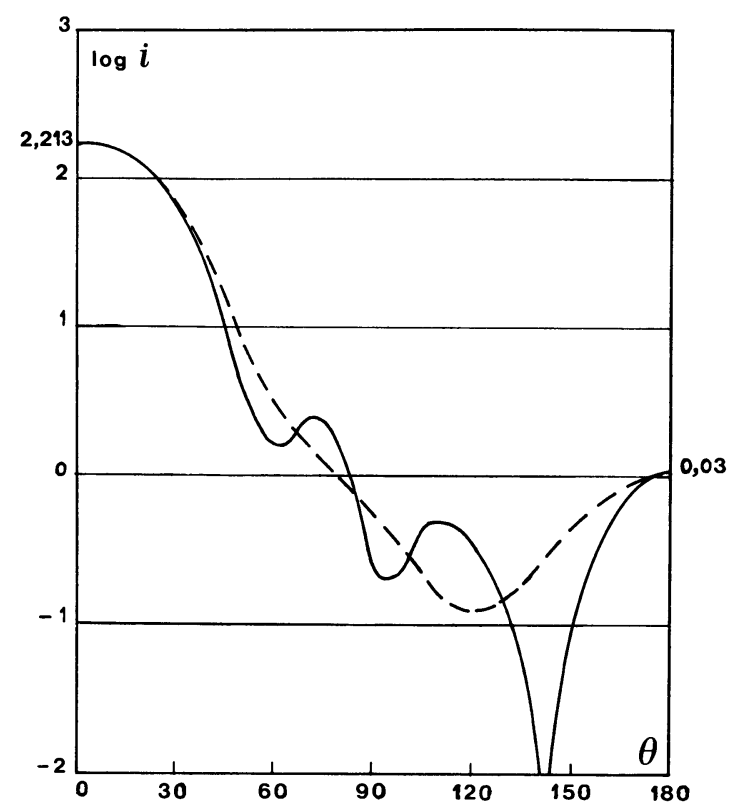

Fig. 3. - Courbes des intensités $i_{1}(\theta)$ (trait plein) et $i_{2}(\theta)$ en fonction de l'angle de diffraction $\theta$ pour $a / b=2 ; n=1,33 ; c=5$ et $\zeta=0^{\circ}$.

[Angular intensity functions $i_{1}(\theta)$ (solid curve) and $i_{2}(\theta)$ plotted against $\theta$ for $a / b=2 ; n=1.33 ; c=5$; $\zeta=0^{\circ}$.]

Quant au problème thermique, qui constitue la seconde phase de cette étude, il consiste à déterminer l'évolution de la température dans le modèle et dans son proche voisinage en considérant l'équation inhomogène de diffusion de la chaleur relativement à un sphéroïde au sein duquel il existe des sources thermiques $Q(\mathbf{r}, t)$ directement liées à la densité de puissance $P_{2}$ précédemment définie. Ceci revient à résoudre l'équation :

$$
\begin{aligned}
\operatorname{div}[k(\mathbf{r}) \operatorname{grad} T(\mathbf{r}, t)]+Q(\mathbf{r}, t) & = \\
& =\rho C_{\mathrm{p}} \partial T(\mathbf{r}, t) / \partial t,
\end{aligned}
$$

pour

$$
\xi<\xi_{0} \text { et } t>0
$$

associée à une condition aux limites inhomogène du troisième type, soit :

$$
k(\mathbf{r}) \partial T(\mathbf{r}, t) / \partial n+h T(\mathbf{r}, t)=\text { Cst. , }
$$


pour

$$
\xi=\xi_{0} \text { et } t>0
$$

où :

$k(\mathbf{r})$ est la conductivité thermique ;

$\rho \quad$ est la masse volumique ;

$C_{\mathrm{p}}$ est la chaleur spécifique ;

$h$ est le coefficient d'échange surfacique.

On peut penser que la résolution de ce problème aux valeurs propres soit grandement facilitée par le traitement effectué lors de la phase précédente mais, malgré cet avantage apparent et plutôt que de rechercher une solution analytique, on a opté pour une méthode entièrement numérique. Celle-ci repose sur une discrétisation du volume tumoral et de son proche voisinage en volumes élémentaires construits à partir des surfaces de coordonnées $\xi=$ Cst., $\eta=$ Cst. et $\phi=$ Cst. On admet ensuite que la conductivité thermique varie avec la variable spatiale $\xi$ afin de prendre en compte la valeur du saut (soit : $\left|k_{2}-k_{1}\right|$ ) à la frontière $\xi=\xi_{0}$; en fait, la valeur du saut est partagée linéairement entre quelques surfaces confocales $\xi=\xi_{0} \pm n \mathrm{~d} \xi$, avec $n=0,1,2$, par exemple. Le terme source $Q(\mathbf{r}, t)$, est la somme du terme source proprement dit $P_{2}(\mathbf{r})$, d'origine électromagnétique, et précédemment obtenu sous forme numérisée, et d'un terme puits $-V_{\mathrm{s}} T(\mathbf{r}, t)$, qui représente le refroidissement des tissus par l'irrigation capillaire.

Cet ensemble de considérations conduit à réécrire les deux équations précédentes dans le système de l'ellipsoïde de révolution allongé :

$$
\begin{aligned}
k(\xi) \Delta T(\xi, & \eta, \phi, t)+ \\
& +1 / h_{\xi}^{2} \partial T(\xi, \eta, \phi, t) / \partial \xi . \partial k(\xi) / \partial \xi \\
& -V_{\mathrm{s}} T(\xi, \eta, \phi, t)+P_{2}(\xi, \eta, \phi) \\
& =\rho C_{\mathrm{p}} \partial T(\xi, \eta, \phi, t) / \partial t
\end{aligned}
$$

$$
k / h_{\xi} \partial T(\xi, \eta, \phi, t) / \partial \xi+h T(\xi, \eta, \phi, t)=0
$$

où $h_{\xi}$ est le coefficient métrique relatif à la variable $\xi$.

Le maillage géométrique est complété par un échantillonnage sur le temps et les expressions différentielles sont remplacées par des différences finies. Si $m$ désigne l'indice lié à la discrétisation de la variable temporelle, alors l'équation précédente s'écrit :

$$
\begin{array}{r}
\left(T^{m+1}-T^{m}\right)=g\left(A T^{m+1}-V_{\mathrm{s}} T^{m}+P\right), \\
\text { avec } g=\delta t / \rho C_{\mathrm{p}}
\end{array}
$$

où : $A$ est une matrice carrée $(n \times n), T$ et $P$ sont des matrices colonnes $(1 \times n), n$ représentant le nombre de points du maillage spatial et $\delta t$ indiquant l'intervalle de temps ; la matrice $A$ tient compte du Laplacien, des conditions aux limites et du terme du premier ordre.

\section{Traitement numérique.}

Plusieurs facteurs conditionnent sa réussite dont, entre autres, le choix du maillage des coordonnées spatiales et temporelle adopté pour résoudre l'équation aux différences finies. On a retenu 31 valeurs pour $\xi$ (les 10 premières correspondant au feuilletage interne du sphéroïde), 19 pour $\eta$ et 9 pour $\phi$ (qui a été limité à varier dans $[0, \pi / 2]$ du fait d'une incidence axiale); il y a donc 5301 points pour les coordonnées spatiales! Pour le temps, on a pris $\delta t=10 \mathrm{~s}$. Des questions de stabilité ont imposé une méthode implicite de résolution, ce qui revient à résoudre l'équation transformée :

$$
T^{m+1}=[1-g \cdot A]^{-1}\left[T^{m}-g \cdot V_{\mathrm{s}} \cdot T^{m}+g \cdot P\right] .
$$

\section{Résultats.}

Les valeurs numériques des différents paramètres ont été empruntées à la littérature spécialisée [12-15] et on a adopté pour la conductivité thermique, $k_{2}$, de la tumeur une valeur quatre fois supérieure à celle, $k_{1}$, du tissu sain environnant [16].

Le rapport $a / b$, qui est une mesure de l'ellipticité du modèle, a une influence marquée sur la répartition du champ électrique réfracté ${ }^{t} \mathbf{E}_{2}$ et donc sur la distribution interne de source $P_{2}$; on peut s'en convaincre en examinant les figures 4,5 et 6 où sont données, dans le plan $\mathrm{O} y z$, les cartes de $\left|{ }^{\mathrm{t}} \mathbf{E}_{2}\right|^{2}$ pour des valeurs du rapport $a / b$ respectivement égales à 1,$67 ; 2 ; 2,22$ et pour une conductivité électrique constante : $\sigma_{2}=0,37 \mathrm{~S} \cdot \mathrm{m}^{-1}$. Le rôle du rapport $a / b$ est également évident sur la figure 7 qui représente les variations de la valeur moyenne de $\left|{ }^{t} \mathbf{E}_{2}\right|^{2}$ et où apparaissent des effets de résonance marqués. Quant à la conductivité électrique $\sigma_{2}$, qui intervient directement dans l'expression de la partie

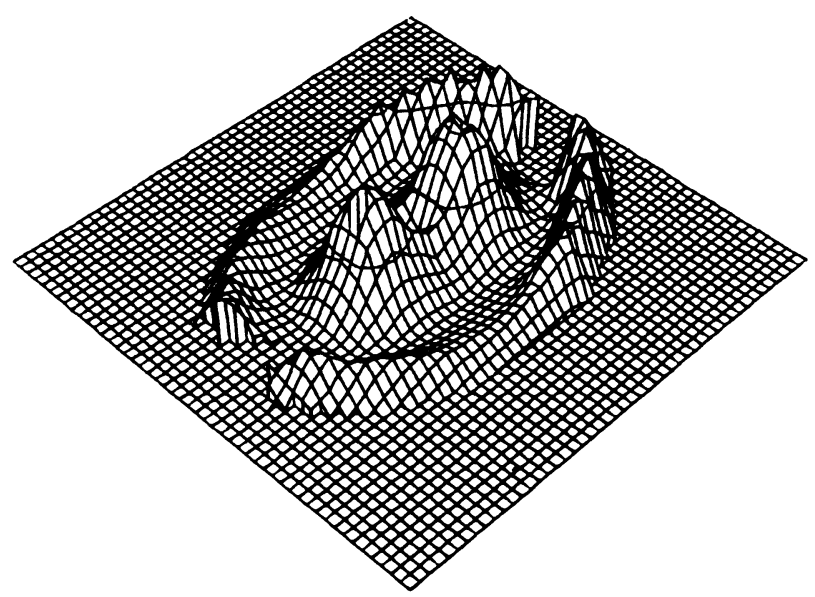

Fig. 4. - Carte de $\left|{ }^{t} \mathbf{E}_{2}\right|^{2}$ pour $a / b=1,67$ et $\sigma_{2}=0,37 \mathrm{~S} \cdot \mathrm{m}^{-1}$.

[Map of $\left|{ }^{t} \mathbf{E}_{2}\right|^{2}$ for $a / b=1.67$ and $\sigma_{2}=0.37 \mathrm{~S} . \mathrm{m}^{-1}$.] 


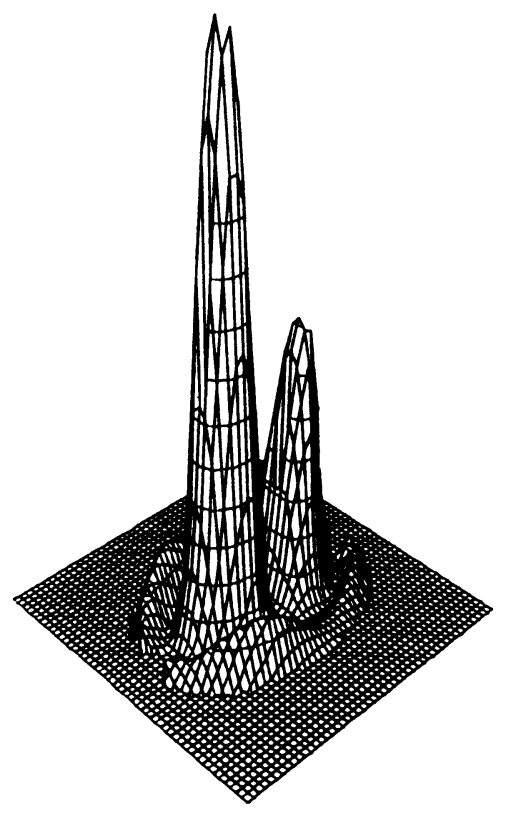

Fig. 5. - Carte de $\left|{ }^{t} \mathbf{E}_{2}\right|^{2}$ pour $a / b=2$ et $\sigma_{2}=0,37 \mathrm{~S} \cdot \mathrm{m}^{-1}$.

[Map of $\left|{ }^{t} \mathbf{E}_{2}\right|^{2}$ for $a / b=2$ and $\sigma_{2}=0.37$ S.m ${ }^{-1}$.]

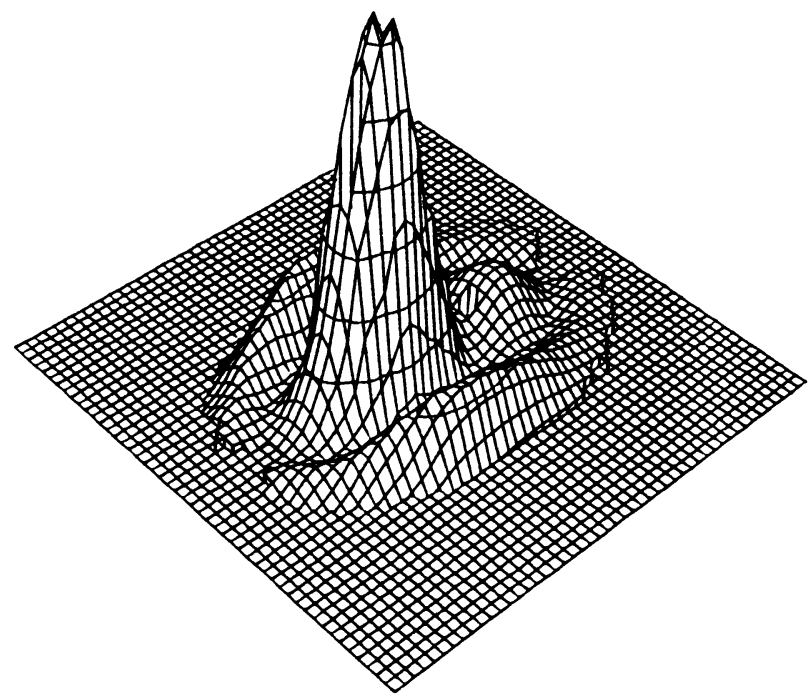

Fig. 6. - Carte de $\left|{ }^{\mathrm{t}} \mathbf{E}_{2}\right|^{2}$ pour $a / b=2,22$ et $\sigma_{2}=0,37 \mathrm{~S} . \mathrm{m}^{-1}$.

[Map of $\left|{ }^{t} \mathbf{E}_{2}\right|^{2}$ for $a / b=2.22$ and $\sigma_{2}=0.37 \mathrm{~S} . \mathrm{m}^{-1}$.]

imaginaire de l'indice complexe, son rôle est également important comme on peut le vérifier en comparant la figure 5 à la figure 8 où, ayant conservé $a / b=2$, on a pris $\sigma_{2}=0,74 \mathrm{~S} \cdot \mathrm{m}^{-1}$; on constate, quand $\sigma_{2}$ crô̂t, un affaissement des cartes de $\left|{ }^{t} \mathbf{E}_{2}\right|^{2}$ corrélatif à une moindre pénétration du champ incident dans le sphéroïde.

$\mathrm{Du}$ point de vue thermique, on note l'importance critique des valeurs des conductivités thermiques des tissus considérés en étudiant la cinétique de chauffe.

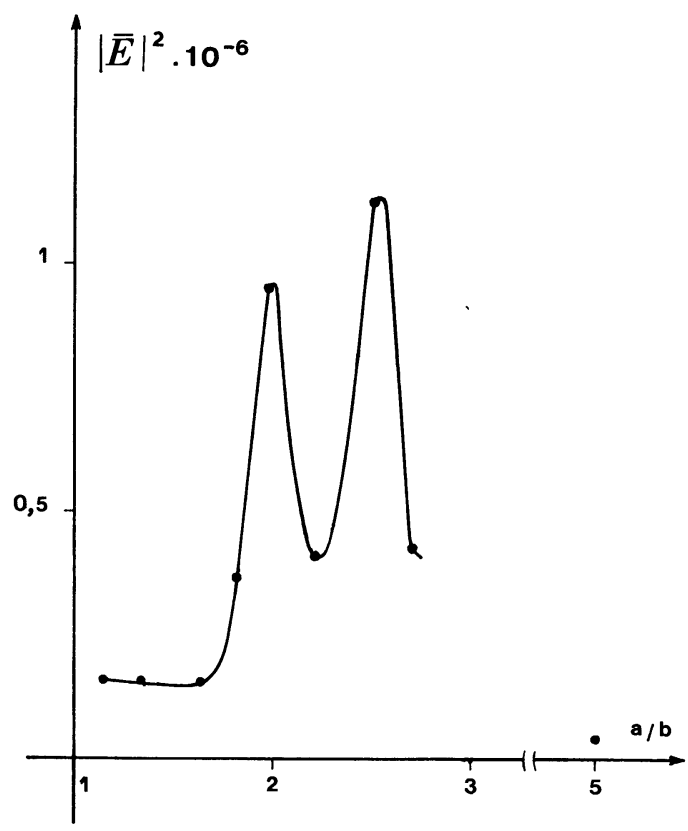

Fig. 7. - Valeur moyenne de $\left|{ }^{t} \mathbf{E}_{2}\right|^{2}$ en fonction de $a / b$.

[Mean value of $\left|{ }^{t} \mathbf{E}_{2}\right|^{2}$ versus $a / b$.]

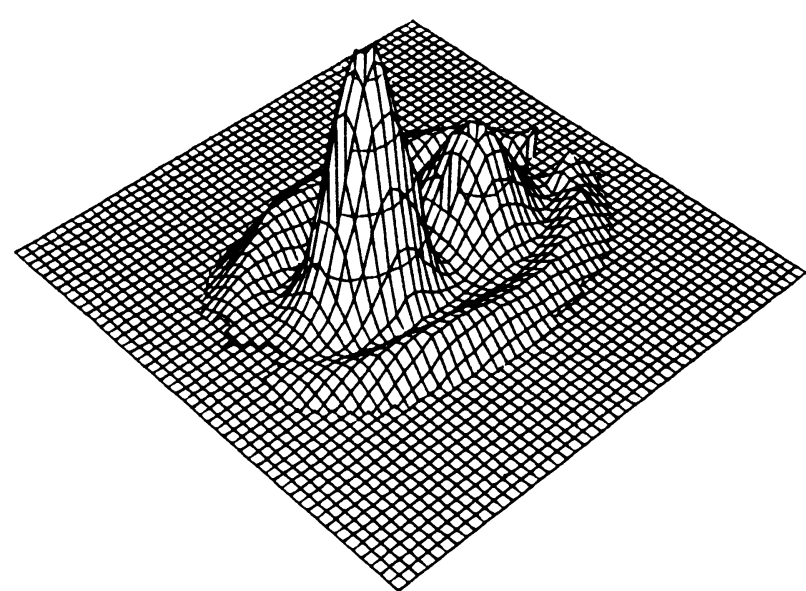

Fig. 8. - Carte de $\left|\mathbf{E}_{2}\right|^{2}$ pour $a / b=2$ et $\sigma_{2}=0,74 \mathrm{~S} . \mathrm{m}^{-1}$.

[Map of $\left|{ }^{t} \mathbf{E}_{2}\right|^{2}$ for $a / b=2$ and $\sigma_{2}=0.74$ S.m ${ }^{-1}$.]

Sur la figure 9 on a représenté, pour des valeurs différentes de $k_{2}$, l'élévation de la température moyenne dans le sphéroïde au cours d'une période de chauffe de 15 min et sa diminution après que le champ électromagnétique appliqué ait cessé d'exister ; les courbes de gauche $\left(k_{2}=0,4 \mathrm{~W} \cdot \mathrm{m}^{-1} \cdot \mathrm{deg}^{-1}\right)$ et de droite $\left(k_{2}=0,04 \mathrm{~W} \cdot \mathrm{m}^{-1} \cdot \mathrm{deg}^{-1}\right)$ ont des allures similaires mais l'élévation de température est d'autant plus rapide que la conductivité est plus faible ; ainsi, l'influence de la conductivité thermique est prépondérante et une détermination correcte de sa valeur in vivo est nécessaire pour envisager une 

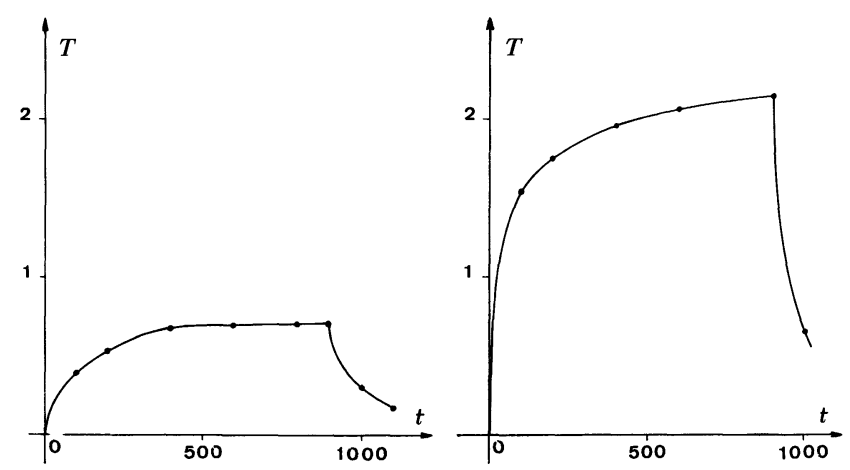

Fig. 9. - Cinétique de chauffe : élévation moyenne de la température dans le sphéroïde durant le chauffage $(0<t \leqslant 900 \mathrm{~s})$ et pendant le refroidissement $(t>900 \mathrm{~s})$ pour deux valeurs de la conductivité thermique : $k_{2}=$ $0,4 \mathrm{~W} \cdot \mathrm{m}^{-1} \cdot \mathrm{deg}^{-1}$ (à gauche) et $k_{2}=0,04 \mathrm{~W} \cdot \mathrm{m}^{-1} \cdot \mathrm{deg}^{-1}$ (à droite). $a=1 \mathrm{~cm} ; a / b=2 ; \sigma_{2}=0,74 \mathrm{~S} \cdot \mathrm{m}^{-1} ; f=4800$ $\mathrm{MHz}$ et $E_{\text {inc }}=1000 \mathrm{~V} \cdot \mathrm{m}^{-1}$.

[Heating kinetic. Mean variation of the temperature inside the spheroid during heating $(0<t \leqslant 900 \mathrm{~s})$ and cooling $(t>900 \mathrm{~s})$ for two values of the thermal conductivity : $k_{2}=0.4 \mathrm{~W} \cdot \mathrm{m}^{-1} \cdot \mathrm{deg}^{-1}$ (left) and $k_{2}=0.04 \mathrm{~W} \cdot \mathrm{m}^{-1} \cdot \mathrm{deg}^{-1}$ (right). $a=1 \mathrm{~cm} ; a / b=2 ; \sigma_{2}=0.74 \mathrm{~S}^{-\mathrm{m}^{-1}} ; f=4800$ $\mathrm{MHz}$ and $E_{\text {inc }}=1000 \mathrm{~V} \cdot \mathrm{m}^{-1}$.

utilisation optimale du modèle à des fins biomédicales. Quant au rapport $a / b$, son influence, sur la distribution de la température, suit pratiquement celle déjà signalée vis-à-vis de la répartition du champ électrique interne $\left|{ }^{t} \mathbf{E}_{2}\right|^{2}$; cette constatation résulte de la relation de proportionnalité existant entre la valeur moyenne du carré du module du champ électrique interne et de l'élévation moyenne de la température dans le sphéroïde (Fig. 10).

Enfin, en ce qui concerne la distribution de la température dans un modèle sphéroïdal de tumeur (en adoptant, par exemple: $f=2450 \mathrm{MHz}$;

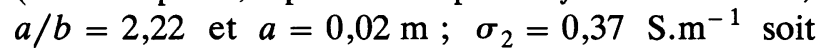
$n_{2}=6+j \cdot 0,22 ;\left|\mathbf{E}_{1}\right|=10 \mathrm{~V} \cdot \mathrm{m}^{-1}$ et $\left.\zeta=0^{\circ}\right)$, on a représenté, pour fixer les idées, les cartes de la température dans le plan $\mathrm{O} x z$ après une exposition de $10 \mathrm{~min}$ au champ électromagnétique micro-onde (Fig. 11) et 2 min après que ce champ ait cessé d'être appliqué (Fig. 12) (l'équidistance des courbes de niveau est de $1^{\circ} \mathrm{C}$ ). La carte de la figure 13 , qui représente la répartition du carré du module du champ électrique interne dans le même plan $\mathrm{O} x z$ peut, par comparaison avec celle de la figure 11, justifier la constatation mentionnée au paragraphe précédent. Ainsi, on observe une concordance assez nette entre la distribution de $\left|{ }^{t} \mathbf{E}_{2}\right|^{2}$ et celle de la température dans le sphéroïde et, surtout, il apparaît qu'une élévation de température de plusieurs degrés peut être enregistrée après une exposition de durée supportable (quelques minutes) à un champ dont l'amplitude n'est pas excessive.

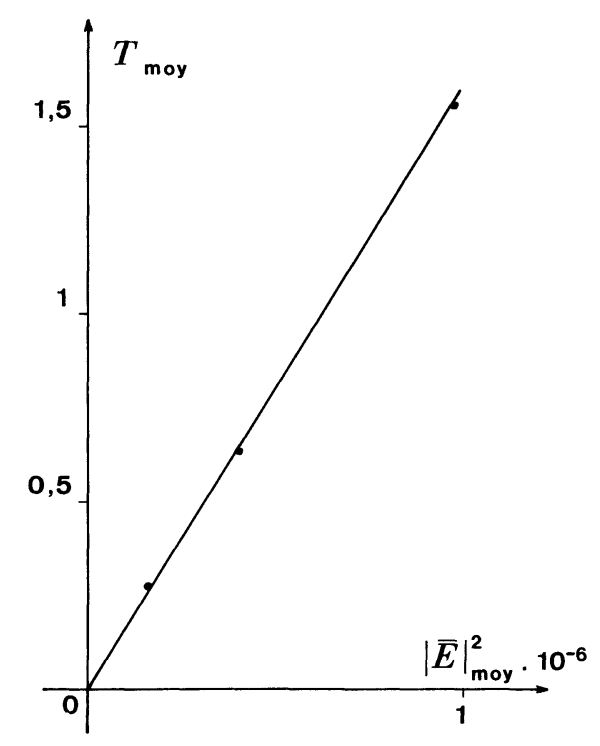

Fig. 10. - Relation entre l'élévation moyenne de la température dans le sphéroïde et $\left|{ }^{t} \mathbf{E}_{2}\right|^{2}$.

[Relation between the mean increase of the temperature inside the spheroid and $\left|{ }^{t} \mathbf{E}_{2}\right|^{2}$.]

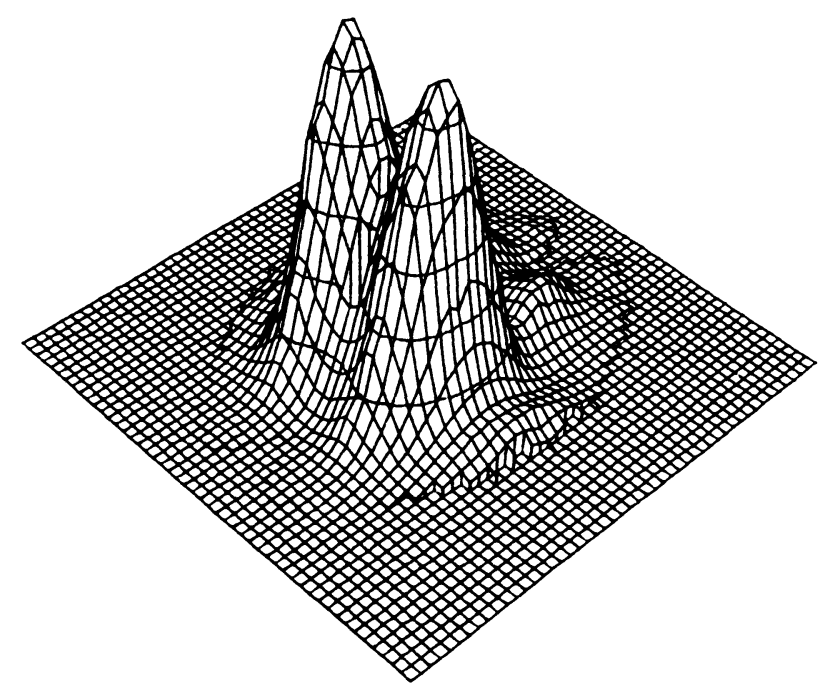

Fig. 11. - Carte de la température dans le plan Oxz après 10 minutes d'exposition au champ micro-onde.

[Typical Map of the temperature in the Oxz plane after 10 min microwave heating.]

\section{Conclusion.}

Les profils de température, au sein d'un modèle sphéroïdal allongé de tumeur soumis à un champ électromagnétique micro-onde, dépendent des paramètres géométriques $a, b, \zeta$ et $\lambda$, mais sont fortement tributaires des valeurs des conductivités thermiques des milieux en présence; celles-ci jouent un rôle capital et conditionnent l'application projetée. Cependant, avec les valeurs admises pour $k_{1}$ et $k_{2}$, et sur la base du modèle adopté, il est possible 
d'envisager positivement l'utilisation des microondes pour provoquer une hyperthermie au sein de ce modèle sphéroïdal de tumeur.

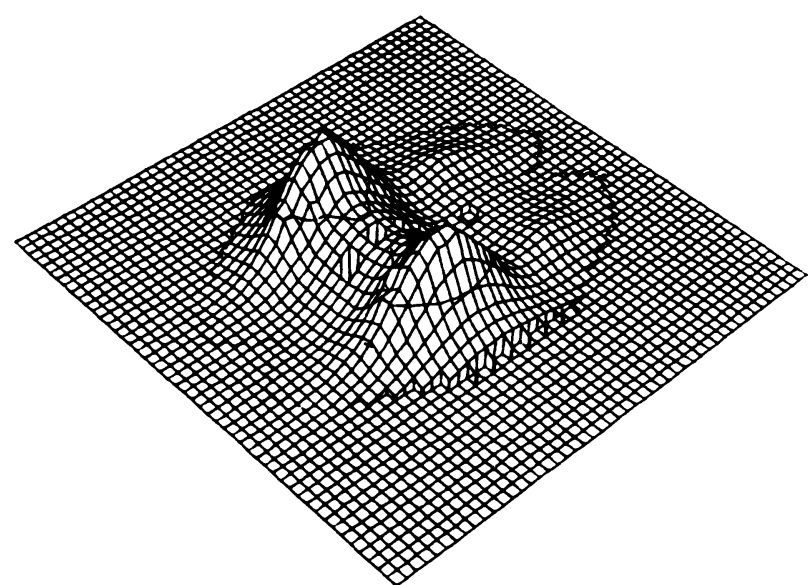

Fig. 12. - Carte de la température dans le plan Oxz 2 minutes après que le champ ait cessé d'être appliqué.

[Map of the temperature in the Oxz plane after $2 \mathrm{~min}$ cooling.]

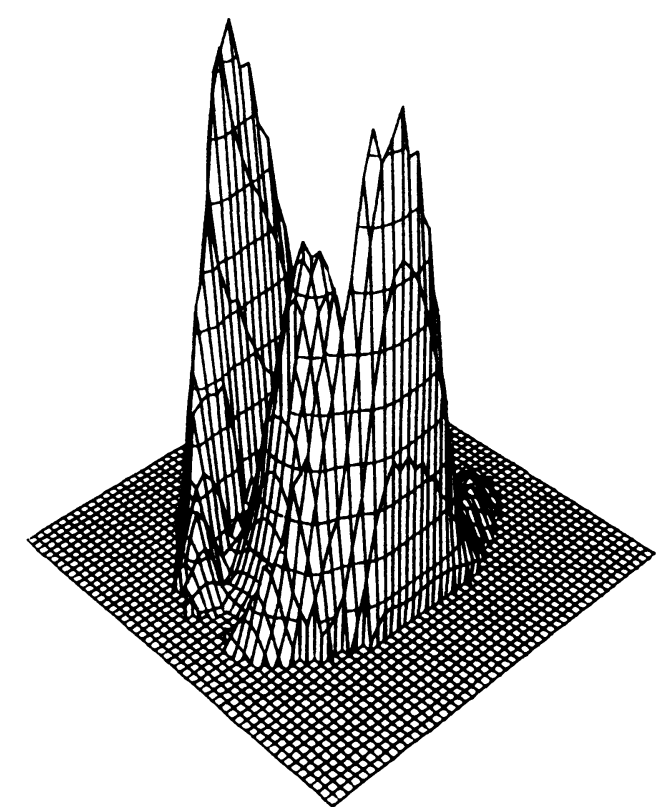

Fig. 13. - Carte de $\left|{ }^{t} \mathbf{E}_{2}\right|^{2}$ dans le plan Oxz correspondant au modèle des figures 11 et 12 .

[Map of $\left|{ }^{t} \mathbf{E}_{2}\right|^{2}$ in the Oxz plane for the previous model.]

\section{Bibliographie}

[1] Braun, J., HaHn, G. M., Enhanced cell killing bleomycin and $43^{\circ}$ hyperthermia and the inhibition of recovery from potentially lethal damage, Cancer Res. 35 (1975) 2921-2927.

[2] Chen, T. T., Heidelberger, C., Quantitative studies on the malignant transformation of mouse prostate cells by carcinogenic hydrocarbons in vitro, Int. J. Cancer 4 (1969) 166-178.

[3] HaHN, G. M., Metabolic aspect of the role of hyperthermia in mammalian cell inactivation and their possible relevance to cancer treatment, Cancer Res. 34 (1974) 3117-3123.

[4] Hill, S. A., Fowler, B. N., Combined effect of heat and X-rays on two types of mouse tumors, 2nd Intern. Symp. on cancer therapy by hyperthermia and radiation (Essen, Germany) June 1977.

[5] Johnson, H. A., Pavelec, M., Thermal enhancement of Thio-Tepa cytotoxity, J. Nat. Cancer Inst. 50 (1973) 903-908.

[6] LI, G. C., SHIU, E., HAHN, G. M., Interaction of $43^{\circ} \mathrm{C}$ hyperthermia and polyene antibiotics : amphotericin B, Lagosin and Filipin, 2nd Intern. Symp. on cancer therapy by hyperthermia and radiation (Essen, Germany) June 1977.

[7] Robinson, J. E., Wizenberg, M. J., McCready, W. A., Radiation and hyperthermal response of normal tissue in situ, Radiobiology 113 (1974) 195-198.

[8] Stratton, J. A., Electromagnetic theory (McGrawHill Book Company, New York) 1941.
[9] Flammer, C., Spheroidal wave functions, Standford U.P. Standford Ca, 1957.

[10] Asano, S., Yамамото, G., Light scattering by a spheroidal particle, Appl. opt. 14, 1 (1975) 2949.

[11] SChaEfer, R. W., Calculations of the light scattered by randomly oriented ensembles of spheroids of size comparable to the wavelength, $\mathrm{Ph}$. $\mathrm{D}$ Thesis State University New York (Albany) 1980.

[12] Burdette, E. C., Cain, F. L., Seals, J., In vivo probe measurement technique for determining dielectric properties at VHF through microwave frequencies, IEEE-MTT 28 (1980) 414-427.

[13] Chan, A. K., Sigelmann, R. A., GuY, A. W., LEHMANN, J. F., Calculation by the method of finite differences of the temperature distribution in layered tissues, IEEE-BME 20 (1973) 86-90.

[14] Kritikos, H. N., SchwaN, H. P., The distribution of heating potential inside lossy spheres, IEEEBME 22 (1975) 457-463.

[15] Stuchly, M. A., STUChly, S. S., Dielectric properties of biological substances, Tabulated 15 (1980) 1.

[16] Gautherie, M., Quenneville, Y., Gros, Ch. M., Thermogénèse des épithéliomas mammaires. III. Etude par fluvographie de la conductibilité thermique des tissus mammaires et de l'influence de la vascularisation tumorale. Biomédecine 22 (1975) 237-245. 